\title{
Developmental PCB Exposure Disrupts Synaptic Transmission and Connectivity in the Rat Auditory Cortex, Independent of Its Effects on Peripheral Hearing Threshold
}

\author{
Christopher M. Lee, ${ }^{1}$ Renee N. Sadowski, ${ }^{1}$ Susan L. Schantz, ${ }^{1,3}$ and ${ }^{-D}$ Daniel A. Llano ${ }^{1,2}$
}

https://doi.org/10.1523/ENEURO.0321-20.2021

${ }^{1}$ Beckman Institute, University of Illinois at Urbana-Champaign, Urbana, IL 61801, ${ }^{2}$ Department of Molecular and Integrative Physiology, University of Illinois at Urbana-Champaign, Urbana, IL 61801, and ${ }^{3}$ Department of Comparative Biosciences, College of Veterinary Medicine, University of Illinois at Urbana-Champaign, Urbana, IL 61802

\begin{abstract}
Polychlorinated biphenyls (PCBs) are enduring environmental toxicants and exposure is associated with neurodevelopmental deficits. The auditory system appears particularly sensitive, as previous work has shown that developmental PCB exposure causes both hearing loss and gross disruptions in the organization of the rat auditory cortex. However, the mechanisms underlying PCB-induced changes are not known, nor is it known whether the central effects of PCBs are a consequence of peripheral hearing loss. Here, we study changes in both peripheral and central auditory function in rats with developmental PCB exposure using a combination of optical and electrophysiological approaches. Female rats were exposed to an environmental PCB mixture in utero and until weaning. At adulthood, auditory brainstem responses (ABRs) were measured, and synaptic currents were recorded in slices from auditory cortex layer 2/3 neurons. Spontaneous IPSCs (sIPSCs) and miniature IPSCs (mIPSCs) were more frequent in PCBexposed rats compared with controls and the normal relationship between IPSC parameters and peripheral hearing was eliminated in PCB-exposed rats. No changes in spontaneous EPSCs were found. Conversely, when synaptic currents were evoked by laser photostimulation of caged-glutamate, PCB exposure did not affect evoked inhibitory transmission, but increased the total excitatory charge, the number and distance of sites that evoke a significant response. Together, these findings indicate that early developmental exposure to PCBs causes long-lasting changes in both inhibitory and excitatory neurotransmission in the auditory cortex that are independent of peripheral hearing changes, suggesting the effects are because of the direct impact of PCBs on the developing auditory cortex.
\end{abstract}

Key words: auditory cortex; laser photostimulation; patch-clamp; PCB; toxin; uncaging

\section{Significance Statement}

The mechanisms by which developmental exposure to polychlorinated biphenyls (PCBs) disrupt the central nervous system are not yet known. Here, we show that developmental PCB exposure is associated with long-lasting dysregulation of both excitatory and inhibitory neurotransmission in the rodent brain. We further find that, unlike controls, synaptic parameters in the auditory cortex of PCB-exposed rats are independent of peripheral hearing changes. These data suggest that PCB-related changes in the auditory cortex are independent of their effects on the auditory periphery and that PCB exposure may disrupt the plastic mechanisms needed to restore normal processing in the auditory cortex after peripheral hearing loss.

Received July 17, 2020; accepted January 6, 2021; First published January 15, 2021.

The authors declare no competing financial interests.
Author contributions: C.M.L., R.N.S., S.L.S., and D.A.L. designed research; C.M.L. and R.N.S. performed research; C.M.L. and R.N.S. analyzed data; C.M.L., R.N.S., S.L.S., and D.A.L. wrote the paper. 


\section{Introduction}

Polychlorinated biphenyls (PCBs) are a family of compounds originally manufactured for many applications, including dielectrics, hydraulic fluids, coolants, and lubricants. PCBs are composed of a biphenyl molecule with chlorine substitutions at any of the ten positions on the biphenyl molecule, creating up to 209 possible congeners. The physical properties and biological effects of PCBs depend on the positions and number of chlorine substitutions. Although their manufacture in the US was banned in 1978, they persist in the environment, and bioaccumulate and biomagnify in food chains, especially in aquatic species, because of their resistance to degradation and their lipophilicity. Additionally, PCBs are transferred to the fetus and infant through the placenta and breast milk (Agency for Toxic Substances and Disease Registry, 2000; for review, see Crinnion, 2011).

Humans and rodents exposed to PCBs experience auditory dysfunction, including higher sound detection thresholds (Goldey et al., 1995; Grandjean et al., 2001; Powers et al., 2006; Trnovec et al., 2008; Min et al., 2014; Li et al., 2015), loss of outer hair cells (Crofton et al., 2000), reduced otoacoustic emission amplitudes (Lasky et al., 2002; Powers et al., 2006; Trnovec et al., 2008), and increased susceptibility to and severity of audiogenic seizures (Poon et al., 2015; Bandara et al., 2016). Complex auditory behaviors such as precise sound localization (Lomber and Malhotra, 2007), temporal processing (Threlkeld et al., 2008), and frequency discrimination of complex stimuli (Znamenskiy and Zador, 2013) require auditory cortical processing in mammals. Developmental exposure to PCBs alters the physiology of the auditory cortex, including delayed auditory P300 latencies (Vreugdenhil et al., 2004), disrupted tonotopic organization of receptive fields (Kenet et al., 2007), and increased sensitivity to GABA blockade (Sadowski et al., 2016). However, the synaptic mechanisms underlying these changes are not known. In addition, it is unclear to what degree these changes are because of direct actions of PCBs in the brain, or whether these changes are secondary effects of peripheral hearing loss.

Hearing loss, when experimentally induced by high level sound exposure, cochlear ablation, or administration of an ototoxic agent, drives plasticity in central auditory structures, weakening inhibitory connections, strengthening excitatory connections, and increasing excitability and spontaneous firing, and these changes generally occur

This work was supported by National Institute of Environmental Health Sciences Grants NIEHS-R01 ES015687 (to S.L.S.) and NIEHS-T32 ES007326 (to C.M.L.), a Beckman Institute Postdoctoral Fellowship (R.N.S.), and funding from the University of Illinois Research Board.

Acknowledgements: We thank Alex Asilador for assistance with speaker calibration and Mindy Howe for help with dosing and breeding.

Correspondence should be addressed to Daniel A. Llano at d-Ilano@ illinois.edu.

https://doi.org/10.1523/ENEURO.0321-20.2021

Copyright @ 2021 Lee et al.

This is an open-access article distributed under the terms of the Creative Commons Attribution 4.0 International license, which permits unrestricted use, distribution and reproduction in any medium provided that the original work is properly attributed. over the course of weeks (Bledsoe et al., 1995; Vale and Sanes, 2002; Wang et al., 2002; Kotak et al., 2005; Sarro et al., 2008; Yang et al., 2012; Chambers et al., 2016; Balaram et al., 2019). These changes effectively increase the gain of the central auditory system and may serve a homeostatic role in restoring central auditory processing after a loss of sensory input (Noreña, 2011; Zeng, 2013; Chambers et al., 2016). Because PCB exposure elevates hearing thresholds, the central auditory system might be expected to respond by reducing inhibition and increasing gain in central structures. Consistent with these predictions, PCB exposure reduces expression of GAD65 in the inferior colliculus (Bandara et al., 2016). However, in the cortex, GAD65 levels are unaffected and thalamocortical transmission is more vulnerable to GABA antagonism in PCB-exposed rats, suggesting PCB treatment is associated with paradoxically higher background levels of cortical inhibition (Bandara et al., 2016; Sadowski et al., 2016).

Synapses in the supragranular layers of the auditory cortex connect neural circuits responsible for a wide range of auditory processes, including cross-frequency integration, sensory gain, coincidence detection, and cross-modality integration (Jiang et al., 2013; Winkowski and Kanold, 2013; Kato et al., 2015; Meng et al., 2017). Therefore, it is important to examine whether PCB exposure affects synaptic connectivity and transmission, as changes could point to underlying causes of complex auditory deficits. Layer $2 / 3$ neurons receive thalamic input, and cortical inputs from all layers, but are more likely to be connected to nearby inputs from layers 2-4 (Atencio and Schreiner, 2010; Oviedo et al., 2010).

To determine the effects of developmental PCB exposure on cortical synaptic transmission, and whether these changes are related to peripheral hearing loss, we dosed rats with either a $6 \mathrm{mg} / \mathrm{kg} / \mathrm{d}$ PCB oil mixture or a control oil mixture beginning four weeks before breeding and continuing until weaning. Because the properties of PCBs vary among congeners, we studied the effects of an environmentally relevant $\mathrm{PCB}$ congener mixture. Experimental subjects were treated with a PCB mixture that mimics the congener profile found in the Fox River in Wisconsin (Kostyniak et al., 2005). From adult offspring, we recorded auditory brainstem responses (ABRs), and excitatory and inhibitory synaptic currents from layer $2 / 3$ auditory cortical neurons, either in the absence of stimulation (spontaneous and miniature currents), or during laser photostimulation of caged glutamate (evoked currents).

\section{Materials and Methods}

\section{PCB exposure and breeding}

All procedures were approved by our university Institutional Animal Care and Use Committee. Rats were maintained in facilities accredited by the Association for the Assessment and Accreditation of Laboratory Animal Care. All animal handling and data collection were performed by experimenters blinded to treatment group. Experimental design and dosing and breeding time 


\begin{tabular}{c|c|c}
\multicolumn{1}{c}{ Day } & \multicolumn{2}{c}{ Control } \\
\hline 0 & PCB Exposed \\
\hline 28 & \\
\hline P110-518 & Data Collection & Data Collection \\
\hline 77 (P21)
\end{tabular}

Figure 1. Experimental design and summary timeline of PCB treatment. Rows indicate significant experimental timepoints: beginning of dosing (day 0), pairing with male (day 28), parturition (approximately day 56), and weaning (approximately day 77 ).

courses are summarized in Figure 1. Long-Evans rats, $8-10$ weeks of age and of both sexes, were purchased from Envigo, and individually housed in standard polycarbonate cages with woodchip bedding. All rats were fed rat chow (Envigo Teklad rodent diet 8604) and water ad libitum. Females were randomly assigned to control or experimental treatments. Beginning one week after receiving the rats, experimental subjects were orally dosed with a PCB mixture in a corn oil vehicle $(6 \mathrm{mg} / \mathrm{kg} /$ d PCB mixture) and control subjects were orally dosed with corn oil alone $(0 \mathrm{mg} / \mathrm{kg} / \mathrm{d}$ PCB mixture). Dosing was accomplished by pipetting the PCB mixture or oil $(0.4$ $\mathrm{ml} / \mathrm{kg}$ ) onto one half of a vanilla wafer cookie (Keebler Golden Vanilla Wafers), which were fed to the rats each day. The PCB mixture (35\% Aroclor 1242, 35\% Aroclor $1248,15 \%$ Aroclor $1254,15 \%$ Aroclor 1260 ) was synthesized to mimic the congener profile found in the walleye fish from the Fox River in Wisconsin (Kostyniak et al., 2005). Experimental rats were dosed at $6 \mathrm{mg} / \mathrm{kg} / \mathrm{d}$, as developmental exposure at this concentration is ototoxic, and increases audiogenic seizure incidence and severity, but does not produce overt signs of clinical toxicity (Kostyniak et al., 2005; Powers et al., 2006, 2009; Bandara et al., 2016). After four weeks of PCB exposure, each female rat was paired with an untreated male rat in a hanging wire cage. Upon detection of a sperm plug indicating gestational day 0 , females were removed from males and daily PCB or control dosing continued through gestation and nursing, until weaning. Litters were standardized to eight pups $2 \mathrm{~d}$ following birth (post-natal day [PND] 2), and pups were weaned on PND21. All offspring were housed in pairs or triplets with cage mates of the same sex and same treatment. All data presented in the current study were collected from female subjects (PCB: $n=60$, control: $n=59$ ).

Rats were dosed and bred from 5/13/2015 to 8/9/2015 by R.N.S. and were used to collect spontaneous and miniature excitatory and inhibitory postsynaptic potentials. A second group of rats was dosed and bred from $10 / 10 / 2016$ to $1 / 4 / 2017$ by C.M.L. and was used to collect input maps by laser photostimulation. ABRs were collected from all rats to measure differences in hearing thresholds between treated and control rats.

\footnotetext{
ABRs

ABRs were collected within one week before electrophysiological recording experiments. Rats were anesthetized
}

with ketamine $(100 \mathrm{mg} / \mathrm{kg})$ and xylazine $(3 \mathrm{mg} / \mathrm{kg})$ and placed in a sound-attenuated chamber. White noise bursts and pure tone pips, both of 5-ms duration, were delivered through an electrostatic speaker (ES1, Tucker Davis Technologies) placed $2.5 \mathrm{~cm}$ from the right ear. ABRs were recorded with two subdermal recording electrodes, one placed above the vertex of the skull, one placed behind the right pinna, and one subdermal ground electrode placed at the base of the tail. The electrodes were connected to a $2400 \mathrm{~A}$ extracellular preamplifier and headstage (Dagan Corporation), or a RA16PA preamplifier and collected on an RP2.1 real-time processor (Tucker Davis Technologies). Signals were digitized and averaged across 512 trials, and bandpassed between 50 and $3000 \mathrm{~Hz}$. ABR thresholds were estimated as the lowest sound level producing a peak in the signal at $3-5 \mathrm{~ms}$ following the sound onset.

\section{Electrophysiology}

We investigated synaptic inputs to auditory cortex of both hemispheres with patch clamp electrophysiology in cortical slices. Rats were anesthetized with ketamine and xylazine, and transcardially perfused with an ice-cold high-sucrose solution (206 mM sucrose, $26 \mathrm{~mm} \mathrm{NaHCO}_{3}$, $11 \mathrm{~mm}$ glucose, $10 \mathrm{~mm} \mathrm{MgCl}, 2.5 \mathrm{~mm} \mathrm{KCl}, 1.25 \mathrm{~mm}$ $\mathrm{NaH}_{2} \mathrm{PO}_{4}$, and $0.50 \mathrm{~mm} \mathrm{CaCl}$ ). The brain was quickly removed and 300- $\mu$ m-thick coronal slices were prepared and allowed to incubate in an oxygenated incubation solution for $1 \mathrm{~h}$ at $32^{\circ} \mathrm{C}$. Slices containing auditory cortex were identified based on visual comparison to a mouse brain atlas (Paxinos and Franklin, 2004). After incubation, a slice was transferred to a recording chamber and immersed in an oxygenated artificial CSF (aCSF; $126 \mathrm{~mm}$ $\mathrm{NaCl}, 26 \mathrm{~mm} \mathrm{NaHCO}_{3}, 10 \mathrm{~mm}$ glucose, $2.5 \mathrm{~mm} \mathrm{KCl}, 2 \mathrm{~mm}$ $\mathrm{CaCl}_{2}, 2 \mathrm{~mm} \mathrm{MgCl}_{2}$, and $1.25 \mathrm{~mm} \mathrm{NaH}_{2} \mathrm{PO}_{4}$ ) at $32^{\circ} \mathrm{C}$. Pyramidal neurons in layer $2 / 3$ were visualized under DIC microscopy, and whole-cell configuration was achieved with borosilicate glass recording pipettes (pipette resistances of 4-10 M $\Omega$ ) filled with internal solution (117 mM $\mathrm{CsOH}, 117 \mathrm{~mm}$ gluconic acid, $11 \mathrm{~mm} \mathrm{CsCl}, 1.0 \mathrm{~mm} \mathrm{MgCl}$, $0.07 \mathrm{~mm} \mathrm{CaCl}_{2}, 10 \mathrm{~mm}$ HEPES, 0.1 mm EGTA, $2.0 \mathrm{~mm} \mathrm{Na}-$ ATP, and $0.4 \mathrm{~mm} \mathrm{Na-GTP;} \mathrm{with} \mathrm{pH} \mathrm{7.3).} \mathrm{No} \mathrm{minimum}$ threshold was set for viable input resistance. Figure $3 A$ depicts the location of the recording pipette in an example auditory cortical slice. Electrophysiological signals were sampled at $20 \mathrm{kHz}$ on a DigiData $1550 \mathrm{~A}$ A/D converter (Molecular Devices).

While holding the cell in voltage clamp, we recorded spontaneous EPSCs (sEPSCs) and sIPSCs, and miniature EPSCs (mEPSCs) and mIPSCs. IPSCs were recorded with bath application of $20 \mu \mathrm{M}$ DNQX and $10 \mu \mathrm{m}$ CPP, while the membrane potential was clamped at $10 \mathrm{mV}$ with a Multiclamp 700B amplifier. EPSCs were recorded with bath application of $20 \mu \mathrm{M}$ GABAzine while the membrane potential was clamped at $-65 \mathrm{mV}$. IPSCs and EPSCs were recorded in slices from separate rats, avoiding cross-contamination of antagonists. Spontaneous currents were initially recorded for $15 \mathrm{~min}$. Subsequently, 1 $\mu \mathrm{m}$ tetrodotoxin (TTX) was added to the aCSF, and mPSCs were recorded for the next $15 \mathrm{~min}$. 
For both 15-min recordings, we identified time windows, lasting between 5-10 min, for analyzing spontaneous and miniature events. The time windows began at least 5 min after breaking the gigaseal for sampling spontaneous events to allow for diffusion of the internal solution into the cell, or at least $5 \mathrm{~min}$ after beginning TTX application for miniature events to allow for wash in. The time windows were adjusted for each recording to include only a period with a stable $(<10 \%$ change) baseline holding current, input resistance, and capacitance. To determine the input resistance of the membrane, we periodically injected hyperpolarizing voltage pulses $(-10 \mathrm{mV}, 100 \mathrm{~ms}$, one pulse per $20 \mathrm{~s})$, and measured the median current response during the first 5 min of recording. IPSC and EPSC events were detected and quantified using Minianalysis software and detected events. Detection thresholds were set at 6-12 pA, and detected events were manually inspected to minimize sampling of noise or artifacts.

\section{Laser scanning photostimulation (LSPS)}

MNI-caged-L-glutamate (Tocris) releases glutamate with exposure to 300-380 nm light, allowing for temporally and spatially precise uncaging of glutamate with laser photostimulation. We produced spatial maps of input strength in coronal slices of auditory cortex, prepared in the same manner as for sPSCs and mPSCs. To generate input maps, we applied to $150 \mu \mathrm{M}$ MNI-glutamate to the aCSF. UV laser light $(355 \mathrm{~nm}, 100-\mathrm{kHz}$ pulses, DPSS Lasers) was guided to the slice by optical path mirrors and lenses (Thorlabs, Newport), and focused through a $10 \times$ objective (Olympus). The beam intensity was attenuated with an acousto-optical modulator (Gooch and Housego), to deliver $24 \mathrm{~mW}$ light at the slice in $1 \mathrm{~ms}$ pulses. At this power, in a separate set of experiments, laser photostimulation was observed to drive spikes in neurons, under a cell-attached configuration, within a $\sim 50-\mu \mathrm{m}$ radius around the laser spot center, similar to what has been seen using a similar laser stimulation configuration in the mouse auditory cortex (Slater et al., 2019).

Maps of synaptic input amplitude and charge to layer 2/ 3 neurons were produced by LSPS; $50 \mu \mathrm{M}$ QX-314 was added to the internal solution for photostimulation experiments to block voltage-gated sodium channels, and a neuron from layer 2/3 was patched in whole-cell configuration and recorded in voltage clamp. The presence of QX-314 precluded comparison of spiking properties between the two groups. In a subset of experiments, two neurons from layer 2/3 were simultaneously patched and recorded during photostimulation. Using Prairie View software or ePhus, the slice was serially photostimulated in a $32 \times 32$ grid of stimulation sites, with adjacent grid points separated by a $40-\mu \mathrm{m}$ distance, serving as a lower bound on the spatial resolution of our analysis. The grid was aligned to the pial surface of the slice, and oriented along the point on the surface closest to the patched neuron(s). Laser stimulation was pulsed for $1 \mathrm{~ms}$ at each stimulation site, and advanced to the successive stimulation site every second. The sequence of stimulation sites was arranged in a non-neighbor order. Patched neurons were recorded in voltage clamp held at $-65 \mathrm{mV}$ during the $32 \times 32$ photostimulation sequence to produce maps of excitatory input, then recorded in voltage clamp held at $10 \mathrm{mV}$ during the photostimulation sequence to produce maps of inhibitory input. Spontaneous events from between slices used for laser photostimulation were not compared with those obtained from slices without caged glutamate because of the possibility that caged glutamate may alter PSC rates.

\section{LSPS analysis}

We measured the charge, amplitude, and latency of the current response to photostimulation at each site. Current signals recorded during photostimulation were lowpass filtered at $150 \mathrm{~Hz}$. The baseline current, measured as the median during the $100 \mathrm{~ms}$ window preceding the laser onset, was subtracted from the signal, so that all measures are relative to the baseline current. All measures were computed from a $200 \mathrm{~ms}$ analysis window starting at the laser onset. $200 \mathrm{~ms}$ was used to capture the full temporal evolution of the photostimulation response, which often lasts 100-200 ms (Callaway and Katz, 1993; Katz and Dalva, 1994; Shepherd et al., 2003). Charge was computed by half-wave rectifying the current (positive rectification for inhibitory charge, and negative rectification for excitatory charge), and integrating the rectified current during $200 \mathrm{~ms}$ analysis window. Amplitude was measured as the maximum current for inhibitory input, and minimum current for excitatory current. Latency was measured as the first time point of the analysis window in which the current exceeded 10\% amplitude. Current responses qualified as "significant" if their amplitudes exceeded 10 times the standard deviation of the baseline window current measured during the $100 \mathrm{~ms}$ before laser onset.

Maps of input charge and amplitude were constructed by ordering response charge and amplitude measures in two-dimensional arrays according to the photostimulation site. Observed amplitude and charges may include spontaneous currents in the recorded neuron that coincide with photostimulation. We took two approaches to reduce noise introduced by photostimulation-independent currents. First, we smoothed amplitude and charge maps by convolving the maps with a $4 \times 4$ Gaussian kernel with standard deviation of 0.5. A similar approach was employed by Kratz and Manis (2015). Second, we included only charges and amplitudes from sites with significant responses. Thus, group-averaged maps and means were computed by including only significant sites from smoothed maps.

Excitatory responses to glutamate uncaging may arise from two sources. EPSCs may be driven by either: (1) the binding of photo-uncaged glutamate to ionotropic glutamate receptors in the recorded cell membrane, considered "direct" responses; or (2) synaptic transmission from presynaptic neurons driven by uncaged glutamate, considered "synaptic" responses. Synaptic responses were separated from direct responses on the basis that synaptic response latencies are later than $7 \mathrm{~ms}$, and direct responses latencies are earlier than $7 \mathrm{~ms}$, as derived 
empirically, see below. Comparable time windows of direct and synaptic responses have been observed by other studies (Kratz and Manis, 2015; Meng et al., 2017).

\section{Statistical analysis}

We tested the effect of PCB treatment on hearing thresholds, spontaneous synaptic current amplitude and frequency, and photostimulation-evoked synaptic current amplitude, charge, latency, input area, mean input distance, and excitation-inhibition ratios. However, these responses may vary with changes of age and hearing thresholds. Furthermore, we typically recorded from multiple neurons from each subject, and sampled multiple subjects from each litter (spontaneous and miniature currents: 116 neurons, 65 subjects, 24 litters, photostimulation-evoked currents: 46 neurons, 32 subjects, 26 litters), potentially introducing litter effects when comparing the responses of individual neurons. To account for response variance introduced by these factors, we used mixed effects modeling to predict our responses with PCB treatment, age, and noise hearing thresholds as fixed effects and birth litter and subject as grouping variables for random effects. For all response variables tested, we did not find a significant relationship between the response variables and age or hearing threshold. We report the significance of group comparisons for each response variable, as the probability of the slope of the response over PCB treatment, against a Student's $t$ distribution.

Ratios of excitatory to inhibitory charge and amplitude are expressed as a gain in $\mathrm{dB}$, as the logarithmic transform of the observed ratios approximately follows a Gaussian distribution.

$$
\text { Gain }=10 * \frac{1}{1024} * \sum_{n=1}^{1024} \log _{10}\left(\frac{R_{e, n}}{R_{i, n}}\right) \text {. }
$$

Here, $R_{e, n}$ and $R_{i, n}$ are the inhibitory and excitatory charge or amplitude response to photostimulation at site $n$ (total stimulation sites $=1024$ ), respectively.

To quantify differences in the spatial profiles of synaptic input, input charge was binned by stimulation site distance in $80-\mu \mathrm{m}$ bins. Mean charge within each bin, across all subjects in each treatment group, was computed. Connection probability was computed as the number of sites with significant responses within each $80-\mu \mathrm{m}$ bin, divided by the total number of sites within that bin.

\section{Results}

\section{Hearing thresholds are elevated for PCB-exposed subjects}

Two separate cohorts of rats were exposed to either a PCB mixture $(6 \mathrm{mg} / \mathrm{kg} / \mathrm{d})$, or a corn oil vehicle, starting at gestation and continuing until weaning. We recorded ABRs to assess hearing thresholds during adulthood (110-518d), and used a mixed-effects model to test the effect of PCB exposure on hearing threshold, independent of age and litter effects. A modest but significant hearing loss was seen with developmental PCB exposure, consistent with previous studies (Powers et al., 2006, 2009). We observed these hearing threshold differences in both rats used for comparing spontaneous and miniature synaptic currents (study 1), and those used to compare photostimulation-evoked currents (study 2). $\mathrm{PCB}$-exposed rats had on average $9.0 \mathrm{~dB}$ higher $\mathrm{ABR}$ thresholds to white noise bursts relative to controls in the first study $\left(t_{(33)}=6.86, p<0.001\right)$, and 5.8-dB higher thresholds in the second $\left(t_{(29)}=4.31, p<0.001\right)$. ABRs to tone pips revealed that $\mathrm{PCB}$ exposure elevated thresholds to $4-$ and $8-\mathrm{kHz}$ tones in both studies, and elevated thresholds to $16-\mathrm{kHz}$ tones in the second study (Fig. 2). Therefore, we confirmed that the PCB-exposed subjects in our study had elevated hearing thresholds, consistent with previous findings.

\section{sIPSCs and mIPSCs are more frequent with PCB exposure}

We asked whether developmental PCB exposure would change inhibitory and excitatory synaptic input to the auditory cortex. To answer this question, we patched layer $2 / 3$ neurons from coronal slices of the auditory cortex and measured sEPSCs or sIPSCs in separate sets of recordings (see Fig. 3 for an example of slice image and sEPSCs). On average, cortical neurons in PCB-exposed subjects received more frequent $\left(t_{(38)}=3.83, p<0.001\right)$ and larger amplitude $\left(t_{(38)}=2.70, p=0.010\right)$ sIPSCs for PCB-exposed subjects compared with controls (Fig. 4A). In contrast, no difference was apparent in the frequency or amplitude of sEPSCs between the two treatment groups. To clarify the potential mechanisms of the synaptic changes, we isolated miniature synaptic currents with bath application of $1 \mu \mathrm{m}$ TTX. Consistent with our observations of spontaneous synaptic currents, PCB treatment was associated with higher mIPSC frequency $\left(t_{(28)}=2.66, p=0.013\right)$, and not associated with differences in mEPSC frequency or amplitude. However, the amplitude of mIPSCS was not different between treatment groups $\left(t_{(28)}=1.59, p=0.12\right)$, suggesting that changes in inhibition with PCB exposure may be mediated primarily by presynaptic changes. Furthermore, input resistance was not affected by PCB exposure (PCB exposed: 188.2 $\pm 34.9 \mathrm{M} \Omega$, control: $225.4 \pm 41.7 \mathrm{M} \Omega, t_{(66)}=1.29$, $p=0.21$ ), suggesting that the increased sIPSC amplitudes are mediated by synaptic mechanisms, rather than changes in the intrinsic membrane properties of cortical neurons. Together, these data point to an increase of spontaneous inhibitory input to layer $2 / 3$ auditory cortex in PCB-exposed subjects.

Changes of inhibitory and excitatory input to auditory cortex following peripheral hearing loss has been well documented (Kotak et al., 2005; Sarro et al., 2008; Balaram et al., 2019). Therefore, changes of inhibition seen in exposed subjects could be a secondary effect of the hearing threshold differences induced by PCB exposure. Among control subjects, increases of hearing threshold predicted reductions of sIPSC frequency $(r=$ -0.44, $p=0.04$ ) and amplitude $(r=-0.42, p=0.04$; Fig. $4 B$, blue points), and reductions of mIPSC frequency ( $r=$ $-0.58, p=0.01)$. These data indicate that in control 
A
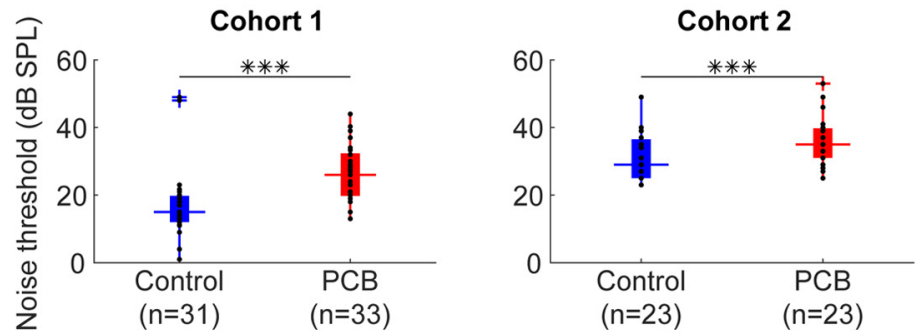

B

\section{Cohort 1}

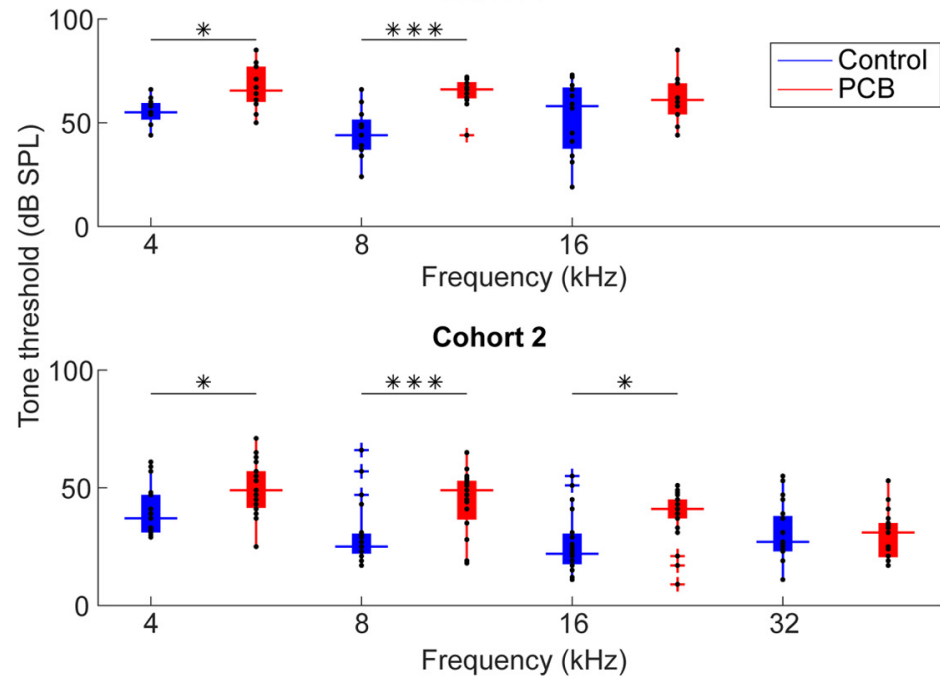

Figure 2. Comparison of ABR thresholds. $A$, Comparison of $A B R$ thresholds in response to noise between control (blue) and PCB (red) treatments. Boxplots indicate median (horizontal bar), 25th and 75th percentiles (box), range of non-outlier points (vertical whiskers), and outliers (crosses). Black asterisks indicate significant comparisons; ${ }^{*} p<0.05,{ }^{* \star *} p<0.001$. $\boldsymbol{B}$, Comparison of thresholds to 4-, 8-, 16-, and 32-kHz tones.

subjects, the auditory system can adjust cortical inhibition to the hearing sensitivity of the animal. However, this relationship was abolished in PCB-exposed subjects (sIPSC frequency: $r=0.15, p=0.55$, amplitude: $r=-0.13$, $p=0.59$, mIPSC frequency: $r=-0.02, p=0.94$, mIPSC amplitude: $r=-0.03, p=0.92$ ), suggesting that the PCBinduced increases in cortical inhibition are not caused by peripheral hearing loss. In contrast to the relationship of hearing thresholds and synaptic inhibition, hearing thresholds did not correlate with SEPSC frequency in either control or PCB-exposed rats (control: $r=0.007$, $p=0.97$ PCB: $r=0.10, p=0.55$ ), amplitude (control: $r=$ $-0.03, p=0.84$, PCB: $r=0.22, p=0.21$ ), or mEPSC frequency (control: $r=0.29, p=0.11$ PCB: $r=-0.21$, $p=0.21$ ) or amplitude (control: $r=-0.052, p=0.79$ PCB: $r=0.17, p=0.39$ ).

\section{LSPS reveals maps of synaptic input to layer $2 / 3$ auditory cortical neurons}

LSPS of caged glutamate allows spatially and temporally precise stimulation of the slice. By using LSPS during recordings of photostimulation-evoked synaptic currents, spatial maps of synaptic strength can be generated (Fig. $5 A-C)$. We determined the optimal time window to separate direct from synaptic inputs by examining the distribution of IPSC latencies. IPSCs measured in this preparation can only be derived via synaptic interactions, and it was found that the distribution of latencies had an inflection point at $7 \mathrm{~ms}$ (Fig. 5D, black arrow). Thus, EPSC latencies shorter than $7 \mathrm{~ms}$ were categorized as direct, while latencies at $7 \mathrm{~ms}$ or longer were categorized as synaptic. To elucidate changes in auditory cortical connectivity associated with PCB exposure, we examined the spatial profiles of synaptic input to layer $2 / 3$ auditory cortex in exposed and control subjects. Figure 6 summarizes the spatial maps produced with LSPS. Here, we align all spatial maps to the recorded neuron, with the pial surface in the positive y direction, and dorsomedial in the positive $x$ direction. Significant responses from stimulation site with the same positions relative to the recorded neuron are averaged across all neurons from each treatment group, and the averaged responses from each relative position are combined to produce the spatial maps. In excitatory maps, responses with a latency $<7 \mathrm{~ms}$ are excluded, to minimize the influence of direct responses to glutamate.

Among maps from control subjects, the strongest excitatory input (Fig. 6, red pixels) is on average near or superficial to the recorded neuron, with minimal excitatory input 


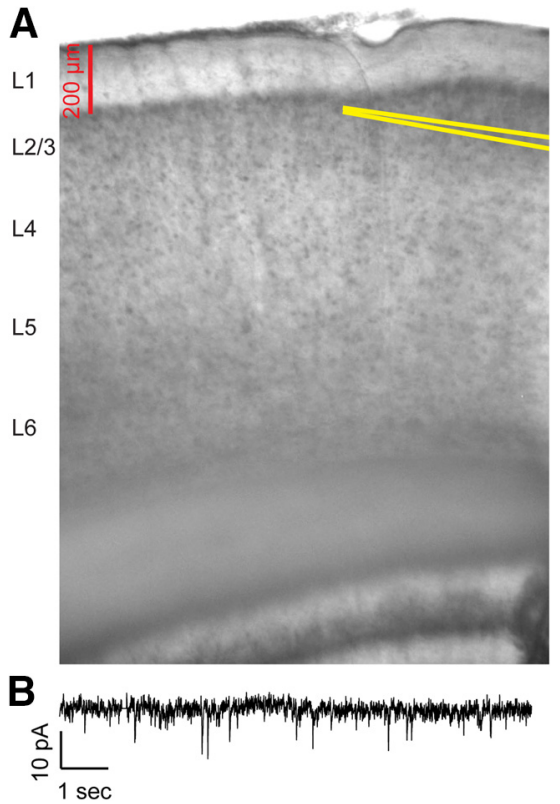

Figure 3. Image of auditory cortex slice and example voltageclamp recording. $\boldsymbol{A}$, Example image of recording electrode placement in a coronal slice containing auditory cortex. Recording pipette walls are highlighted in yellow lines. $\boldsymbol{B}$, Example of membrane current recorded in voltage clamp with holding potential of $-65 \mathrm{mV}$ and bath application of 20 $\mu \mathrm{m}$ GABAzine.

(depicted in dark blue pixels) from any point $>400-\mu \mathrm{m}$ distance from the recorded cell. In maps from PCB-exposed subjects, the strongest excitatory input is also near or superficial to the recorded neuron, with moderate input (light blue to cyan pixels) spanning most of the photostimulation map. Inhibitory maps for both control and PCB-exposed subjects were similar in shape. The strongest inhibitory input was near the recorded neuron, and average inhibitory strength drops off rapidly with increasing distance.

\section{PCB-exposed cells receive more total excitatory input charge, and more distant excitatory inputs}

We estimated the total synaptic input for each recorded cell by summing the amplitudes and charges evoked from all stimulation sites that yielded a significant response (Fig. $7 A, B$, first two columns). Cells from control subjects received $0.12 \pm 0.03 \mathrm{nC}$ of total excitatory charge and $2.07 \pm 0.72 \mathrm{nC}$ of total inhibitory charge. Cells from PCBexposed subjects received $0.31 \pm 0.10 \mathrm{nC}$ of total excitatory charge and $2.10 \pm 1.10 \mathrm{nC}$ of total inhibitory charge. PCB exposure was associated with a significant increase in excitatory charge $\left(t_{(39)}=2.26, p=0.030\right)$, but no change in inhibitory charge $\left(t_{(39)}=0.029, p=0.98\right)$. However, the total of photostimulation-evoked excitatory and inhibitory current amplitudes were not different between control (excitation: $0.59 \pm 0.20 \mathrm{nA}$, inhibition: $6.40 \pm 2.22 \mathrm{nA}$ ) and PCB-exposed groups (excitation: $1.09 \pm 0.36 \mathrm{nA}$, inhibition: $5.09 \pm 2.28 \mathrm{nA}$, comparison of excitation: $t_{(39)}=1.52$, $p=0.14$, inhibition: $\left.t_{(39)}=0.28, p=0.78\right)$. The ratio of excitation to inhibition was not significantly different between treatments (Charge E/I, control: $-2.1 \pm 1.8 \mathrm{~dB}$, PCB-exposed: $-3.6 \pm 1.2 \mathrm{~dB}, t_{(30)}=0.56, p=0.58 ;$ Amplitude E/I, control: $-0.7 \pm 1.7 \mathrm{~dB}$, PCB-exposed: $-2.2 \pm 0.9 \mathrm{~dB}$, $\left.t_{(30)}=0.52, p=0.61\right)$. In addition, latency was not affected by PCB treatment (excitatory latencies, control: $17.2 \pm 6.4 \mathrm{~ms}$ PCB: $17.2 \pm 9.7 \mathrm{~ms}, t_{(37)}=0.31, p=0.76$, inhibitory latencies, control: $12.4 \pm 7.2 \mathrm{~ms}$, PCB: $12.3 \pm 5.9 \mathrm{~ms}$, $\left.t_{(38)}=0.62, p=0.54\right)$. In summary, relative to controls, PCBexposed cells responded to the excitatory inputs evoked by photostimulation with greater charge, but similar amplitude, and responded similarly to inhibitory inputs.

The difference in total excitatory charge between PCBexposed and control subjects may be because of a change in the number of input sites, or the strength of each input site. We observed that cells from PCB-exposed subjects received significant excitatory input from more sites than cells from controls (control: $17.9 \pm 6.0$ sites, PCB-exposed: $33.8 \pm 9.3$ sites, $p=0.048$ ). On the other hand, the average charge per site was not significantly different between treatments (control: $7.2 \pm 1.3 \mathrm{pA}$, PCB-exposed: $9.0 \pm 1.9 p A, t_{(39)}=0.35, p=0.72$ ). Thus, cortical neurons from $\mathrm{PCB}$-exposed rats receive input from a greater number of sites than controls but receive input of the same strength from each site.

The spatial profile of input strength is plotted as a function of stimulation site distance in Figure 8, which provides a graphical representation of the analysis shown in Figure 7. Differences in excitatory input response charge, and excitatory input site number are most pronounced between $\sim 100-$ and $700-\mu \mathrm{m}$ distance. Furthermore, the average distance of significant excitatory stimulation sites is longer in the PCB-exposed group compared with controls (control: $215 \pm 25.8 \mu \mathrm{m}$, PCB-exposed: $284 \pm 25.3$ $\left.\mu \mathrm{m}, t_{(39)}=2.23, p=0.032\right)$. In contrast, the average distance of significant inhibitory stimulation sites is not different between the groups (control: $239 \pm 32 \mu \mathrm{m}$, PCB: $239 \pm 29 \mu \mathrm{m}, t_{(38)}=0.16, p=0.87$ ). Distances were clustered into layers based on contrast seen on DIC images, and no differences were seen between groups (not shown). Together, these findings suggest that in PCB-exposed subjects, layer $2 / 3$ auditory cortical neurons integrate excitatory input from a greater number of neurons at intermediate $(100-700 \mu \mathrm{m})$ distances, without affecting inhibitory connections.

\section{Discussion}

\section{Developmental PCB exposure induces long-lasting increases in spontaneous inhibitory tone and increases in excitatory connectivity in auditory cortex}

We found that developmental PCB exposure results in paradoxically increased SIPSC amplitude and frequency and increased mIPSC frequency in layer $2 / 3$ of the auditory cortex (Fig. 4A), while also inducing peripheral hearing loss. The increased inhibition appears to be mediated by presynaptic changes, as the amplitude of mIPSCs is unchanged with PCB treatment. In contrast, PCB treatment did not affect photostimulation-evoked IPSCs. Therefore, PCB exposure may induce changes 

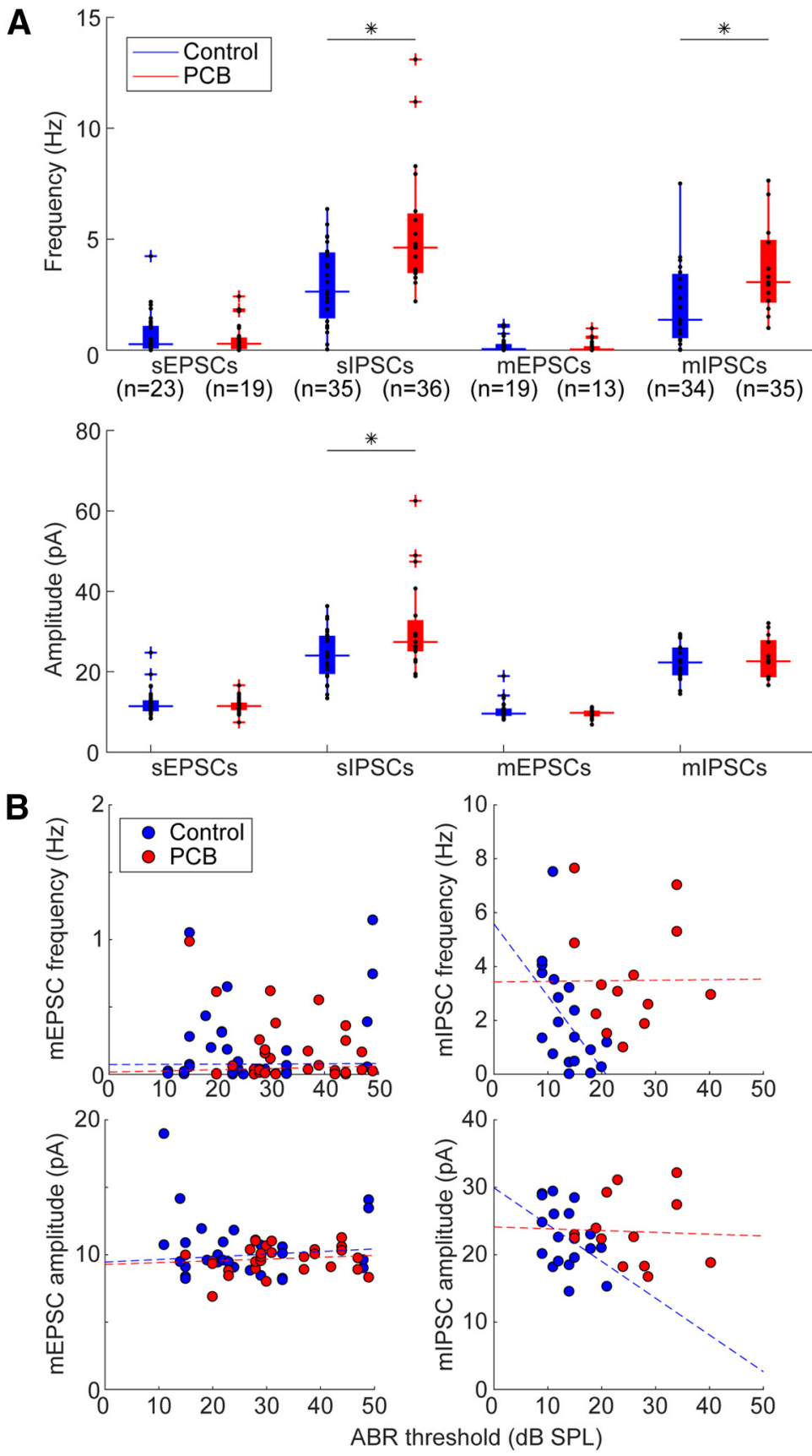

Figure 4. Comparison of spontaneous and miniature synaptic currents. $\boldsymbol{A}$, Comparison of frequency of synaptic currents between control (blue) and PCB-treated (red) neurons (sample sizes indicate numbers of neurons). Boxplots indicate median (horizontal bar), 25th and 75th percentiles (box), range of non-outlier points (vertical whiskers), and outliers (crosses). Black asterisks indicate significant comparisons, ${ }^{*} p<0.05$. B , Relationship of ABR threshold and sIPSC frequency for control (blue points) and PCB-exposed (red points) groups. Dashed lines indicate robust linear regression fits.

that specifically affect spontaneous release of GABA from inhibitory cortical neurons, without affecting the strength of evoked inhibitory synaptic transmission. While evoked synaptic currents depend on synaptic density and efficacy of each synapse, spontaneous synaptic transmission reflects synaptic efficacy and rate of vesicle release. Therefore, $\mathrm{PCB}$ exposure may increase vesicle release of inhibitory inputs. Vesicle release can be modified by a variety of changes, including changes of activity, membrane potential, intracellular calcium dynamics, and the readily releasable pool. Increases of spontaneous inhibition following developmental PCB exposure are consistent with previous findings that thalamocortical transmission is more strongly enhanced by $\mathrm{GABA}_{\mathrm{A}}$-receptor blockade in PCB-exposed subjects (Sadowski et al., 2016). Because PCB 
A

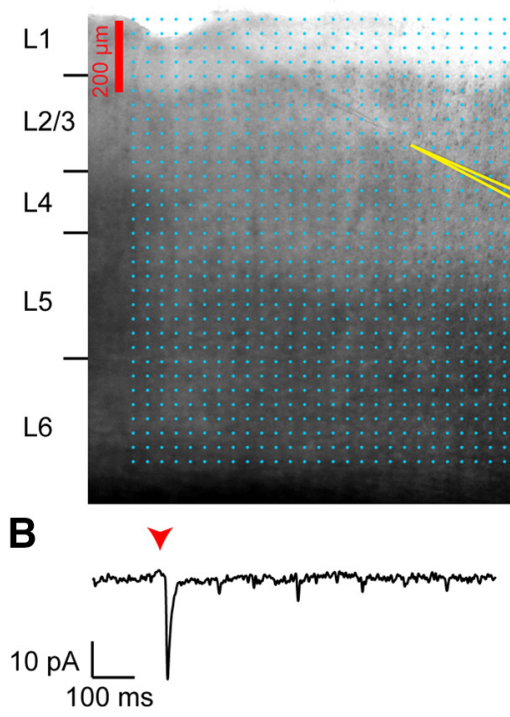

C

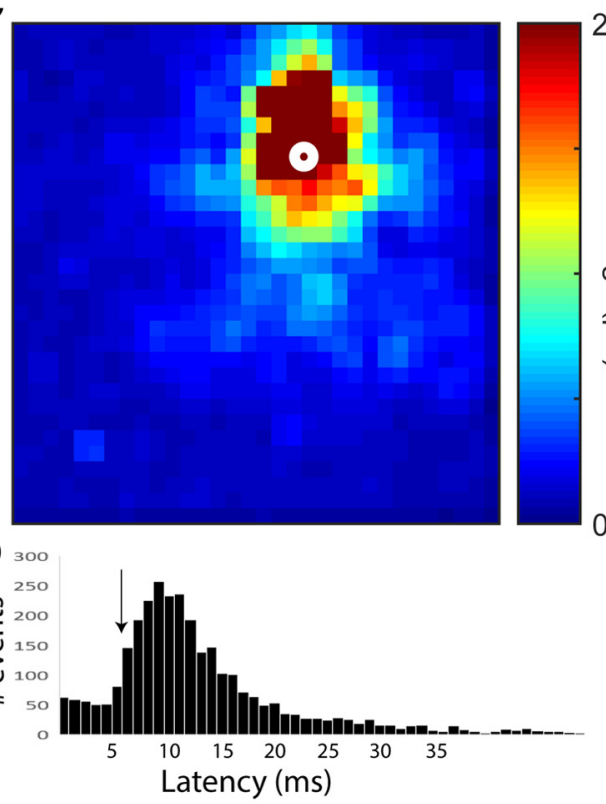

20

Figure 5. Demonstration of LSPS mapping of input charge. $\boldsymbol{A}$, Example image demonstrating positions of stimulation grid and recording electrodes in a coronal slice containing auditory cortex. Cyan points mark the sites of the $32 \times 32$ photostimulation grid. Recording pipette walls are highlighted in yellow lines. In the example, current recordings were simultaneously collected from two neurons. $\boldsymbol{B}$, An example photostimulation-evoked current response from the cell positioned on the bottom right. Holding potential was $-65 \mathrm{mV}$. Timing of the laser pulse (1 ms in duration) is indicated by the red arrowhead. A pronounced negative peak begins shortly after the laser onset. C, Map of input charge from current responses to photostimulation at all sites of the $32 \times 32$ stimulation grid. For recordings of excitatory responses, measured charge is inverted to positive values, and represented by color. $\boldsymbol{D}$, Distribution of all IPSC latencies across all neurons in this study. The black arrow corresponds to the latency used to distinguish between direct and synaptic events in this study.

exposure increases spontaneous inhibitory input to auditory cortical neurons, release from inhibition with $\mathrm{GABA}_{\mathrm{A}}$-receptor antagonist application is more pronounced in PCB-exposed subjects compared with controls.

It is important to note that rats used to study photostimulation-evoked currents had unexpectedly higher white noise thresholds and lower pure tone thresholds than those used to study spontaneous and miniature currents $\left(t_{(106)}=6.84, p<0.001\right)$. The reasons for these differences are not known, but may include: (1) evaluation of $A B R$ thresholds by different experimenters; (2) different headstage and preamplifiers used between studies (study 1 : Dagan 2400A, study 2: TDT RA4LI/RA4PA); or (3) true hearing threshold differences between the different cohorts of rats, purchased approximately two years apart. Nonetheless, PCB exposed rats showed significantly higher noise, $4-\mathrm{kHz}$ tone, and $8-\mathrm{kHz}$ tone thresholds in each study.

Developmental PCB exposure does not affect the frequency and amplitude of sEPSCs or mEPSCs (Fig. 4A), suggesting that synaptic transmission of individual synapses and spontaneous excitatory input do not change with PCB exposure. However, in neurons from PCB-exposed subjects, EPSCs were evoked from a larger number of photostimulation sites, and they were evoked from more distant sites on average (Fig. 7A). Thus, rather than changing the strength of individual synapses, PCB exposure results in abnormally enhanced connectivity between excitatory cortical neurons, with the largest changes at distances of $100-700 \mu \mathrm{m}$ (Fig. 8). Because layer $2 / 3$ cortical neurons integrate input from neurons with different frequency tuning, PCB-induced changes in excitatory connectivity may disrupt frequency receptive fields in auditory cortex (Kenet et al., 2007). Together, these changes suggest that PCB exposure may degrade spectral resolution as a result of excessive excitatory connectivity in the cortex.

Among the control subjects, higher ABR thresholds are associated with reduced cortical inhibition, which may reflect a compensatory increase of gain in the central auditory system (Fig. 4B). However, with PCB exposure, inhibition is not related to hearing threshold. Therefore, PCB exposure may disrupt the compensatory regulation of cortical activity by modulation of central auditory gain seen in unexposed subjects. Furthermore, PCB exposure increases spontaneous inhibitory input in the cortex, while elevating hearing thresholds. Thus, while PCB exposure impairs hearing, its effects on spontaneous inhibition would paradoxically further reduce activity in the auditory cortex, potentially compounding auditory perceptual deficits.

\section{Potential mechanisms of changes}

Increases of SIPSC amplitude and frequency seen in PCB-exposed subjects were unexpected and contrast 
A Control $(n=23)$
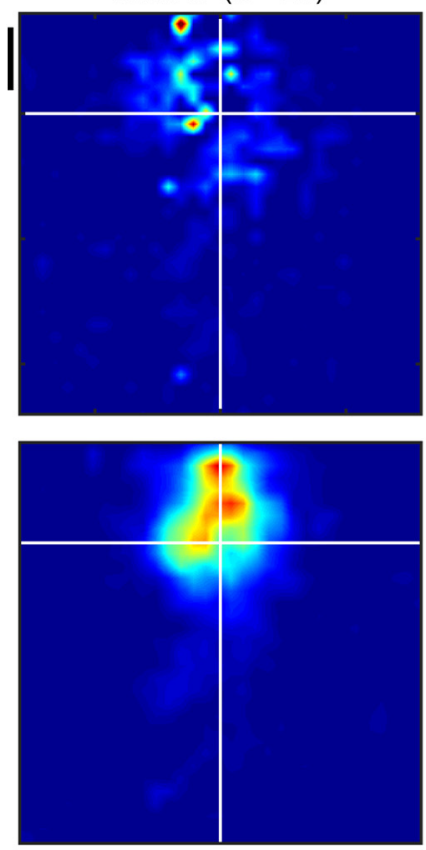

B
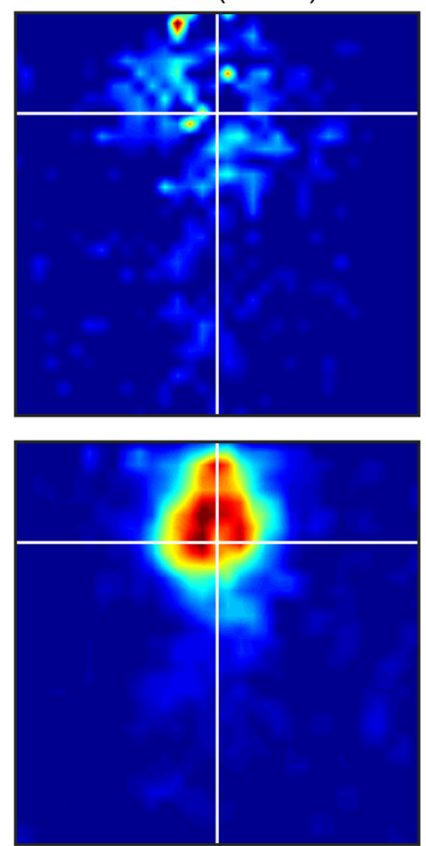

PCB $(n=23)$
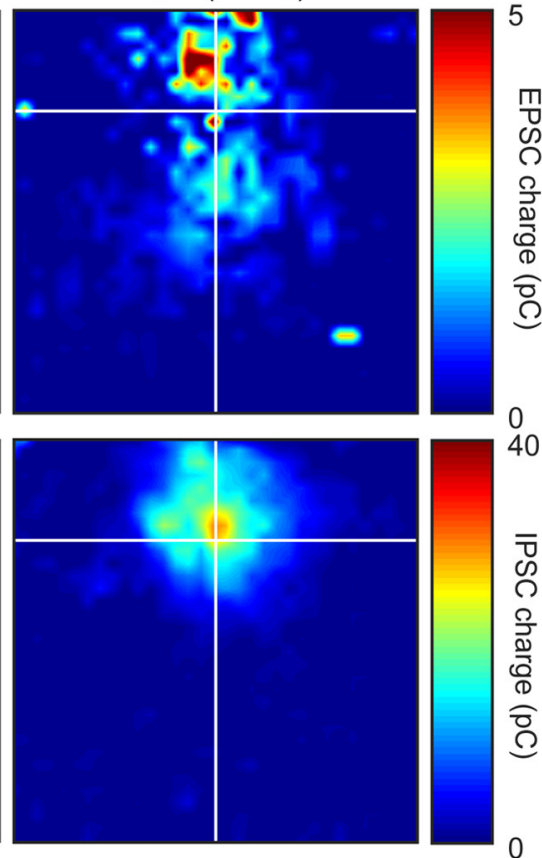

PCB $(n=23)$
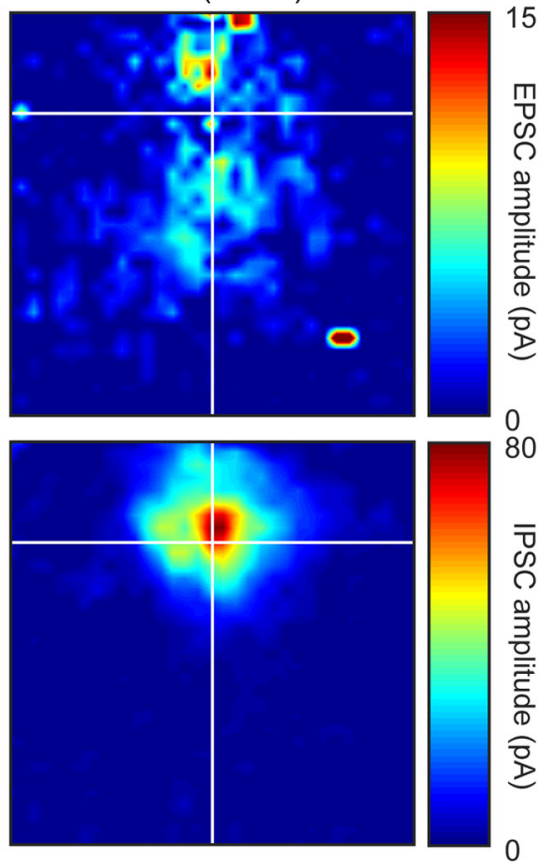

Figure 6. Group averaged maps of photostimulation-evoked synaptic strength. Photostimulation-evoked input maps of charge (A) and amplitude $(\boldsymbol{B})$ aligned to the recorded cell body. Measured charge at each site is averaged across all cells from each treatment group and is represented in color. EPSC charge maps are presented in the top plots, and IPSC charge maps are presented in the bottom plots. Black vertical scale bar marks $200 \mu \mathrm{m}$.

previous findings of reduced central inhibition following hearing loss (Bledsoe et al., 1995; Vale and Sanes, 2002; Kotak et al., 2005; Sarro et al., 2008; Balaram et al., 2019). Several differences between PCB-induced hearing loss and aforementioned studies may explain differences in the outcomes. First, the hearing impairment following $\mathrm{PCB}$ exposure is relatively mild, elevating thresholds by
$<10 \mathrm{~dB}$ on average as observed in the current study and in previous studies (Powers et al., 2006, 2009). Exposure to $\mathrm{PCBs}$ leads to a loss of outer hair cells and reduced otoacoustic emissions (Crofton et al., 2000; Lasky et al., 2002; Powers et al., 2006; Trnovec et al., 2008). Inner hair cells, on the other hand, are spared after exposure to a commercial PCB mixture (Aroclor 1254; Crofton et al., 
A Excitation
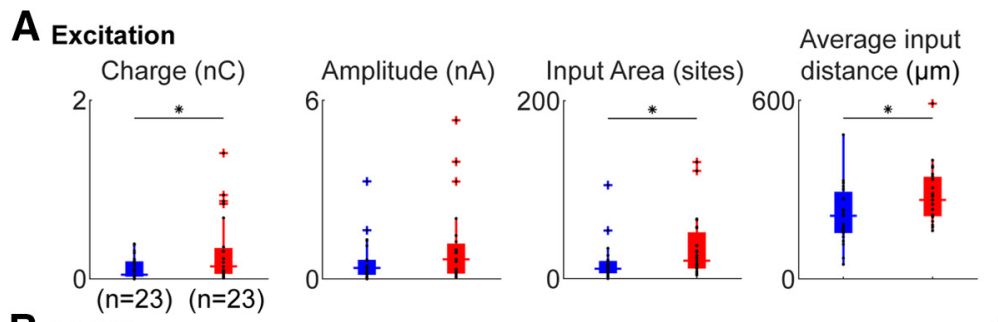

C

B Inhibition
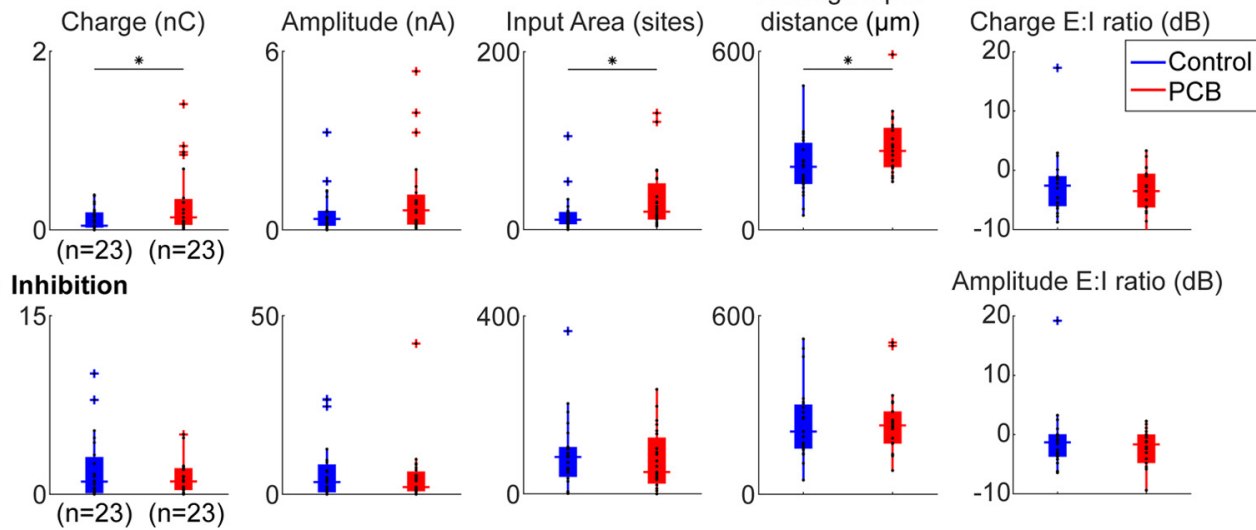

Amplitude $\mathrm{E}: \mathrm{I}$ ratio $(\mathrm{dB})$

Figure 7. Comparison of photostimulation-evoked currents between treatments. Comparison of total excitatory $(\boldsymbol{A})$ and inhibitory $(B)$ input charge, input area, and input distance, between control (blue) and PCB-exposed (red) treated neurons. Boxplots indicate median (horizontal bar), 25th and 75th percentiles (box), range of non-outlier points (vertical whiskers), and outliers (crosses). Black asterisks indicate significant comparisons; ${ }^{*} p<0.05$. $\boldsymbol{C}$, Ratio of excitatory to inhibitory charge between control and PCB-exposed groups.

2000), but it is not yet known whether they are affected by the Fox River PCB mixture. PCBs and other dioxin-like compounds reduce thyroid hormone levels, and this thyroid hormone deficiency may be involved in PCB-induced hearing loss, as thyroxine replacement partially restores hearing in PCB-exposed animals (Goldey et al., 1995; Goldey and Crofton, 1998; Poon et al., 2011). Furthermore, developmental hypothyroidism can affect the development, connectivity, and organization of auditory cortical neurons (Ruiz-Marcos et al., 1983; Berbel et al., 1993;
Lucio et al., 1997). In contrast, studies documenting increases of central auditory gain following hearing loss typically involve damage to inner hair cells and threshold increases of $30 \mathrm{~dB}$ or more. Therefore, PCB-induced hearing loss may involve specific mechanisms not typically seen with peripheral hearing loss, that result in increased cortical inhibition.

Second, in addition to damaging the sensory epithelium, PCBs also have direct actions in the central nervous system. The Fox River PCB mixture used in this study
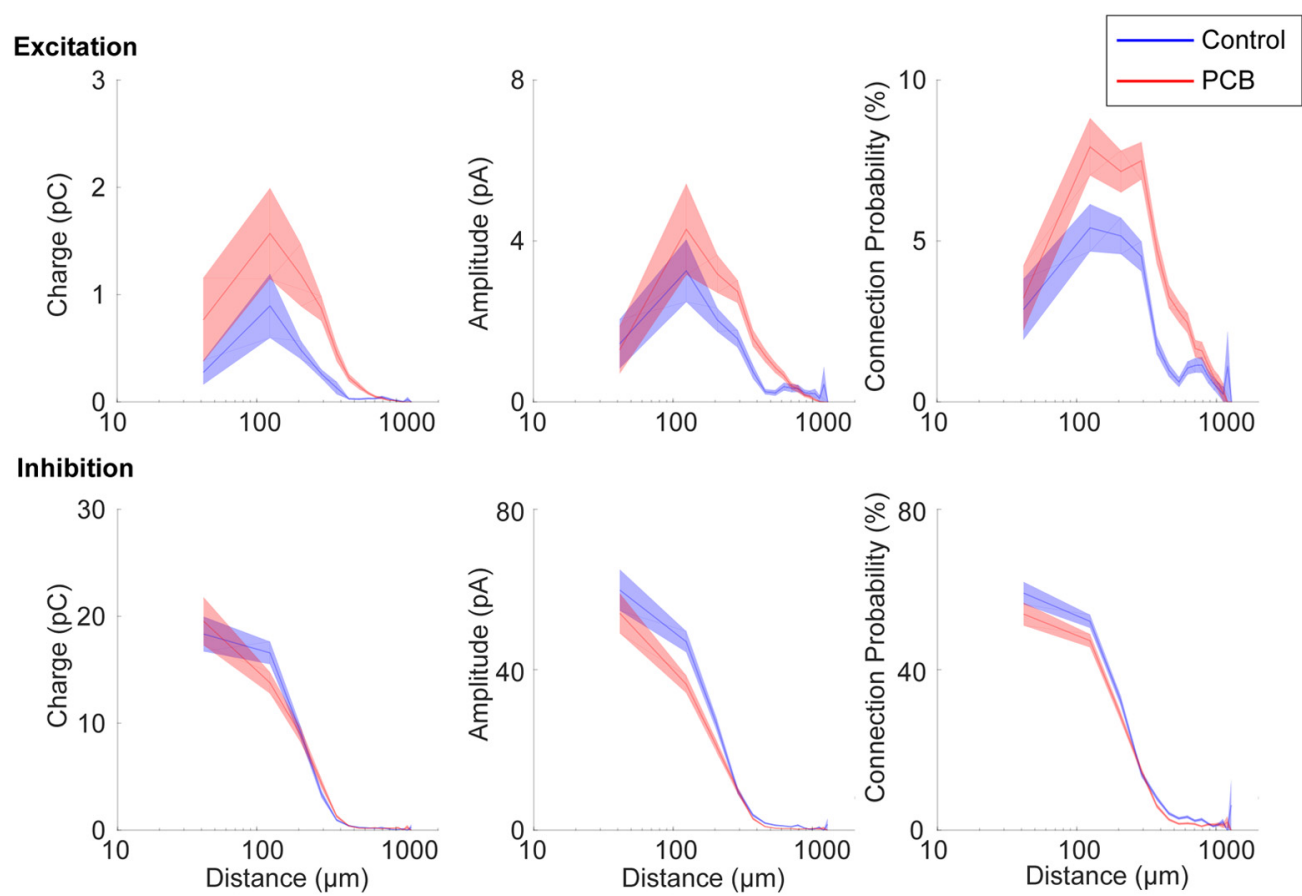

Figure 8. Spatial profile of synaptic input. Distance profile of input charge and connection probability for control (blue) and PCB-exposed (red) treatment groups. Response measures are binned by input distance in $80-\mu \mathrm{m}$ bins, and interpolation between bin means are marked by the solid lines. The shaded areas indicate 1 standard error bounds around the means. 
was previously found to increase binding of ryanodine to ryanodine receptors (RyRs; Kostyniak et al., 2005). Ryanodine receptor activation can induce growth of dendrites, and RyR-dependent increases in dendritic growth have been observed following developmental exposure to PCBs (Lein et al., 2007; Yang et al., 2009). Furthermore, developmental PCB exposure disrupts both experiencedependent synaptic plasticity and Morris water maze learning, supporting the idea that the activation of RyRs may underlie some of the behavioral effects of PCBs (Yang et al., 2009). PCB exposure not only affects dendritic growth, but also alters excitatory and inhibitory synaptic transmission in auditory cortex and hippocampus (Kenet et al., 2007; Kim et al., 2009). In hippocampal slices, changes in synaptic transmission following wash-in of PCBs were found to be dependent on RyR activation (Kim et al., 2009). We observed an increase in the number of sites producing significant excitatory synaptic responses to laser photostimulation (Figs. 7A, 8). This change may be explained by increased synaptic connectivity between excitatory cortical neurons because of higher levels of RyR activation in PCB-exposed subjects.

In summary, we find that developmental exposure to PCBs increases spontaneous inhibitory input to the neurons in layer $2 / 3$ of the auditory cortex, increases the number of excitatory connections, and disrupts the relationship between inhibition and hearing impairment. These changes were unexpected as the auditory system typically responds to hearing loss by increasing gain in central auditory structures. Thus, in addition to elevating hearing thresholds, PCB exposure may disrupt plastic changes needed to restore central auditory function after hearing loss by increasing spontaneous cortical inhibition. Thus, the cognitive deficits associated with PCB exposure in humans (Vreugdenhil et al., 2002; Schantz et al., 2003; Newman et al., 2006), may be related to longlasting changes in the underlying synaptic architecture that alter local cortical network connectivity.

\section{References}

Agency for Toxic Substances and Disease Registry (2000) Toxicological profile for polychlorinated biphenyls (PCBs). Washington, DC: United States Department of Health Services, Public Health Service.

Atencio CA, Schreiner CE (2010) Columnar connectivity and laminar processing in cat primary auditory cortex. PLoS One 5:e9521.

Balaram P, Hackett TA, Polley DB (2019) Synergistic transcriptional changes in AMPA and GABA $A_{A}$ receptor genes support compensatory plasticity following unilateral hearing loss. Neurosci 407:108119.

Bandara SB, Eubig PA, Sadowski RN, Schantz SL (2016) Developmental PCB exposure increases audiogenic seizures and decreases glutamic acid decarboxylase in the inferior colliculus. Toxicol Sci 149:335-345.

Berbel P, Guadaño-Ferraz A, Martínez M, Quiles JA, Balboa R, Innocenti GM (1993) Organization of auditory callosal connections in hypothyroid rats. Eur J Neurosci 5:1465-1478.

Bledsoe SC, Nagase S, Miller JM, Altschuler RA (1995) Deafness-induced plasticity in the mature central auditory system. Neuroreport 7:225-229.

Callaway EM, Katz LC (1993) Photostimulation using caged glutamate reveals functional circuitry in living brain slices. Proc Natl Acad Sci USA 90:7661-7665.
Chambers AR, Resnik J, Yuan Y, Whitton JP, Edge AS, Liberman MC, Polley DB (2016) Central gain restores auditory processing following near-complete cochlear denervation. Neuron 89:867879.

Crinnion WJ (2011) Polychlorinated biphenyls: persistent pollutants with immunological, neurological, and endocrinological consequences. Altern Med Rev 16:5-13.

Crofton KM, Ding DL, Padich R, Taylor M, Henderson D (2000) Hearing loss following exposure during development to polychlorinated biphenyls: a cochlear site of action. Hear Res 144:196-204.

Goldey ES, Crofton KM (1998) Thyroxine replacement attenuates hypothyroxinemia, hearing loss, and motor deficits following developmental exposure to Aroclor 1254 in rats. Toxicol Sci 45:94-105.

Goldey ES, Kehn LS, Lau C, Rehnberg GL, Crofton KM (1995) Developmental exposure to polychlorinated biphenyls (Aroclor 1254) reduces circulating thyroid hormone concentrations and causes hearing deficits in rats. Toxicol Appl Pharmacol 135:77-88.

Grandjean P, Weihe P, Burse VW, Needham LL, Storr-Hansen E, Heinzow B, Debes F, Murata K, Simonsen H, Ellefsen P, BudtzJørgensen E, Keiding N, White RF (2001) Neurobehavioral deficits associated with PCB in 7-year-old children prenatally exposed to seafood neurotoxicants. Neurotoxicol Teratol 23:305-317.

Jiang X, Wang G, Lee AJ, Stornetta RL, Zhu JJ (2013) The organization of two novel cortical interneuronal circuits. Nat Neurosci 16:210-218.

Kato HK, Gillet SN, Isaacson JS (2015) Flexible sensory representations in auditory cortex driven by behavioral relevance. Neuron 88:1027-1039.

Katz LC, Dalva MB (1994) Scanning laser photostimulation: a new approach for analyzing brain circuits. J Neurosci Methods 54:205218.

Kenet T, Froemke RC, Schreiner CE, Pessah IN, Merzenich MM (2007) Perinatal exposure to a noncoplanar polychlorinated biphenyl alters tonotopy, receptive fields, and plasticity in rat primary auditory cortex. Proc Natl Acad Sci USA 104:7646-7651.

Kim KH, Inan SY, Berman RF, Pessah IN (2009) Excitatory and inhibitory synaptic transmission is differentially influenced by two orthosubstituted polychlorinated biphenyls in the hippocampal slice preparation. Toxicol Appl Pharmacol 237:168-177.

Kostyniak PJ, Hansen LG, Widholm JJ, Fitzpatrick RD, Olson JR, Helferich JL, Kim KH, Sable HJK, Seegal RF, Pessah IN, Schantz SL (2005) Formulation and characterization of an experimental PCB mixture designed to mimic human exposure from contaminated fish. Toxicol Sci 88:400-411.

Kotak VC, Fujisawa S, Lee FA, Karthikeyan O, Aoki C, Sanes DH (2005) Hearing loss raises excitability in the auditory cortex. J Neurosci 25:3908-3918.

Kratz MB, Manis PB (2015) Spatial organization of excitatory synaptic inputs to layer 4 neurons in mouse primary auditory cortex. Front Neural Circuits 9:1-17.

Lasky RE, Widholm JJ, Crofton KM, Schantz SL (2002) Perinatal exposure to Aroclor 1254 impairs distortion product otoacoustic emissions (DPOAEs) in rats. Toxicol Sci 68:458-464.

Lein PJ, Yang D, Bachstetter AD, Tilson HA, Harry GJ, Mervis RF, Kodavanti PRS (2007) Ontogenetic alterations in molecular and structural correlates of dendritic growth after developmental exposure to polychlorinated biphenyls. Environ Health Perspect 115:556-563.

Li MC, Wu HP, Yang CY, Chen PC, Lamber GH, Guo YL (2015) Gestational exposure to polychlorinated biphenyls and dibenzofurans induced asymmetric hearing loss: Yucheng children study. Environ Res 137:65-71.

Lomber S, Malhotra SG (2007) Sound localization during homotopic and heterotopic bilateral cooling deactivation of primary and nonprimary auditory cortical areas in the cat. J Neurophysiol 97:2643.

Lucio RA, García JV, Ramón Cerezo J, Pacheco P, Innocenti GM, Berbel P (1997) The development of auditory callosal connections in normal and hypothyroid rats. Cereb Cortex 7:303-306. 
Meng X, Kao JPY, Lee HK, Kanold PO (2017) Intracortical circuits in thalamorecipient layers of auditory cortex refine after visual deprivation. eNeuro 4:ENEURO.0092-17.2017-11.

Min JY, Kim R, Min KB (2014) Serum polychlorinated biphenyls concentrations and hearing impairment in adults. Chemosphere 102:6-11.

Newman J, Aucompaugh A, Schell LM, Denham M, DeCaprio AP, Gallo MV, Ravenscroft J, Kao CC, Hanover MR, David D, Jacobs AM, Tarbell AM, Worswick P; Akwesasne Task Force on the Environment (2006) PCBs and cognitive functioning of Mohawk adolescents. Neurotoxicol Teratol 28:439-445.

Noreña AJ (2011) An integrative model of tinnitus based on a central gain controlling neural sensitivity. Neurosci Biobehav Rev 35:1089-1109.

Oviedo HV, Bureau I, Svoboda K, Zador AM (2010) The functional asymmetry of auditory cortex is reflected in the organization of local cortical circuits. Nat Neurosci 13:1413-1420.

Paxinos G, Franklin KBJ (2004) The mouse brain in stereotaxic coordinates. San Diego: Gulf Professional.

Poon E, Powers BE, McAlonan RM, Ferguson DC, Schantz SL (2011) Effects of developmental exposure to polychlorinated biphenyls and/or polybrominated diphenyl ethers on cochlear function. Toxicol Sci 124:161-168.

Poon E, Bandara SB, Allen JB, Sadowski RN, Schantz SL (2015) Developmental PCB exposure increases susceptibility to audiogenic seizures in adulthood. Neurotoxicology 46:117-124.

Powers BE, Poon E, Sable HJ, Schantz SL (2009) Developmental exposure to PCBs, MeHg, or both: long-term effects on auditory function. Environ Health Perspect 117:1101-1107.

Powers BE, Widholm JJ, Lasky RE, Schantz SL (2006) Auditory deficits in rats exposed to an environmental PCB mixture during development. Toxicol Sci 89:415-422.

Ruiz-Marcos A, Salas J, Sanchez-Toscano F, Escobar del Rey F, Morreale de Escobar G (1983) Effect of neonatal and adult-onset hypothyroidism on pyramidal cells of the rat auditory cortex. Dev Brain Res 9:205-213.

Sadowski RN, Stebbings KA, Slater BJ, Bandara SB, Llano DA, Schantz SL (2016) Developmental exposure to PCBs alters the activation of the auditory cortex in response to $\mathrm{GABA}_{\mathrm{A}}$ antagonism. Neurotoxicology 56:86-93.

Sarro EC, Kotak VC, Sanes DH, Aoki C (2008) Hearing loss alters the subcellular distribution of presynaptic GAD and postsynaptic $\mathrm{GABA}_{\mathrm{A}}$ receptors in the auditory cortex. Cereb Cortex 18:28552867.

Schantz SL, Widholm JJ, Rice DC (2003) Effects of PCB exposure on neuropsychological function in children. Environ Health Perspect 111:357-376.
Shepherd GMG, Pologruto TA, Svoboda K (2003) Circuit analysis of experience-dependent plasticity in the developing rat barrel cortex. Neuron 38:277-289.

Slater BJ, Sons SK, Yudintsev G, Lee CM, Llano DA (2019) Thalamocortical and intracortical inputs differentiate layer-specific mouse auditory corticollicular neurons. J Neurosci 39:256-270.

Threlkeld SW, Penley SC, Rosen GD, Fitch RH (2008) Detection of silent gaps in white noise following cortical deactivation in rats. Neuroreport 19:893-898.

Trnovec $T$, Sovčíková $E$, Hust'ák $M$, Wimmerová $S$, Kočan $A$, Jurečková D, Langer P, Palkovičová L, Drobná B (2008) Exposure to polychlorinated biphenyls and hearing impairment in children. Environ Toxicol Pharmacol 25:183-187.

Vale C, Sanes DH (2002) The effect of bilateral deafness on excitatory and inhibitory synaptic strength in the inferior colliculus. Eur $\mathrm{J}$ Neurosci 16:2394-2904.

Vreugdenhil HJI, Lanting $\mathrm{Cl}$, Mulder PGH, Boersma ER, WeisglasKuperus N (2002) Effects of prenatal PCB and dioxin background exposure on cognitive and motor abilities in Dutch children at school age. J Pediatr 140:48-56.

Vreugdenhil HJI, Van Zanten GA, Brocaar MP, Mulder PGH, Weisglas-Kuperus N (2004) Prenatal exposure to polychlorinated biphenyls and breastfeeding: opposing effects on auditory P300 latencies in 9-year-old Dutch children. Dev Med Child Neurol 46:398-405.

Wang J, Ding D, Salvi RJ (2002) Functional reorganization in chinchilla inferior colliculus associated with chronic and acute cochlear damage. Hear Res 168:238-249.

Winkowski DE, Kanold PO (2013) Laminar transformation of frequency organization in auditory cortex. J Neurosci 33:1498-1508.

Yang D, Kim KH, Phimister A, Bachstetter AD, Ward TR, Stackman RW, Mervis RF, Wisniewski AB, Klein SL, Kodavanti PRS, Anderson KA, Wayman G, Pessah IN, Lein PJ (2009) Developmental exposure to polychlorinated biphenyls interferes with experience-dependent dendritic plasticity and ryanodine receptor expression in weanling rats. Environ Health Perspect 117:426-435.

Yang S, Su W, Bao S (2012) Long-term, but not transient, threshold shifts alter the morphology and increase the excitability of cortical pyramidal neurons. J Neurophysiol 108:1567-1574.

Zeng F (2013) An active loudness model suggesting tinnitus as increased central noise and hyperacusis as increased nonlinear gain. Hear Res 295:172-179.

Znamenskiy P, Zador TM (2013) Corticostriatal neurons in auditory cortex drive decisions during auditory discrimination. Nature 497:482-487. 